\title{
The Morningside Initiative: Collaborative Development of a Knowledge Repository to Accelerate Adoption of Clinical Decision Support
}

\author{
Robert Greenes ${ }^{*}, 1$, Meryl Bloomrosen ${ }^{2}$, Nancy E. Brown-Connolly ${ }^{3}$, Clayton Curtis ${ }^{4}$, Don E. Detmer ${ }^{5}$, \\ Robert Enberg ${ }^{6}$, Douglas Fridsma ${ }^{1}$, Emory Fry $^{7}$, Mary K. Goldstein ${ }^{8}$, Peter Haug ${ }^{9}$, Nathan Hulse ${ }^{9}$, \\ Tonya Hongsermeier ${ }^{10}$, Saverio Maviglia ${ }^{10}$, Craig W. Robbins ${ }^{11}$ and Hemant Shah ${ }^{6}$
}

${ }^{1}$ Arizona State University, Phoenix, AZ, USA

${ }^{2}$ American Medical Informatics Association, Bethesda, MD, USA

${ }^{3}$ Telemedicine and Advanced Technology Research Center (TATRC) of the US Army Medical Research Materiel Command, Frederick, MD, USA

${ }^{4}$ Veterans Health Administration, Boston, MA, USA

${ }^{5}$ American Medical Informatics Association, Bethesda, MD, USA

${ }^{6}$ Henry Ford Health System, Detroit, MI, USA

${ }^{7}$ Naval Health Research Center, San Diego, CA, USA

${ }^{8}$ VA Palo Alto Health Care System, Palo Alto, CA, USA

${ }^{9}$ Intermountain Healthcare, Salt Lake City, UT, USA

${ }^{10}$ Partners Healthcare, Boston, MA, USA

${ }^{11}$ Kaiser Permanente-Care Management Institute, Aurora, CO, USA

\begin{abstract}
The Morningside Initiative is a public-private activity that has evolved from an August, 2007, meeting at the Morningside Inn, in Frederick, MD, sponsored by the Telemedicine and Advanced Technology Research Center (TATRC) of the US Army Medical Research Materiel Command. Participants were subject matter experts in clinical decision support (CDS) and included representatives from the Department of Defense, Veterans Health Administration, Kaiser Permanente, Partners Healthcare System, Henry Ford Health System, Arizona State University, and the American Medical Informatics Association (AMIA). The Morningside Initiative was convened in response to the AMIA Roadmap for National Action on Clinical Decision Support and on the basis of other considerations and experiences of the participants. Its formation was the unanimous recommendation of participants at the 2007 meeting which called for creating a shared repository of executable knowledge for diverse health care organizations and practices, as well as health care system vendors. The rationale is based on the recognition that sharing of clinical knowledge needed for CDS across organizations is currently virtually non-existent, and that, given the considerable investment needed for creating, maintaining and updating authoritative knowledge, which only larger organizations have been able to undertake, this is an impediment to widespread adoption and use of CDS. The Morningside Initiative intends to develop and refine (1) an organizational framework, (2) a technical approach, and (3) CDS content acquisition and management processes for sharing CDS knowledge content, tools, and experience that will scale with growing numbers of participants and can be expanded in scope of content and capabilities. Intermountain Healthcare joined the initial set of participants shortly after its formation. The efforts of the Morningside Initiative are intended to serve as the basis for a series of next steps in a national agenda for CDS. It is based on the belief that sharing of knowledge can be highly effective as is the case in other competitive domains such as genomics. Participants in the Morningside Initiative believe that a coordinated effort between the private and public sectors is needed to accomplish this goal and that a small number of highly visible and respected health care organizations in the public and private sector can lead by example. Ultimately, a future collaborative knowledge sharing organization must have a sustainable long-term business model for financial support.
\end{abstract}

Keywords: Clinical decision support, knowledge bases, knowledge sharing.

\section{A. INTRODUCTION}

The Morningside Initiative described herein is a collaboration of participants in public and private health care

*Address correspondence to this author at the Department of Biomedical Informatics, Arizona State University, 425 N. 5th Street, Phoenix, AZ 85004, USA; Tel: 602-827-2548; Fax: 602-827-2564;

E-mail: greenes@asu.edu organizations that has been formed to address the challenges to widespread adoption of computer-based clinical decision support (CDS) through sharing of CDS knowledge content, tools, and experience. The purpose of this paper is to describe the goals of the effort and the approach being taken. 


\section{A.1. Background}

The importance of computer-based clinical decision support (CDS) is based on the many problems facing health care, and the need for proactive, point-of-care, patientspecific approaches to ensure that best practices are adopted [1]. Among the well-recognized problems are: spiraling costs $[2,3]$, disparities of access and large numbers of uninsured $[4,3]$, errors and unevenness of quality [5-8], slow dissemination of advances $[9,10]$, inefficiencies and waste $[11,3]$, fragmentation and poor communication [12,13], and a lack of patient-centered care [6].

CDS has been shown to be useful in fostering patient safety, health care quality, and cost-effectiveness of care [1]. Further, CDS fulfills the practical need of health care delivery organizations to respond effectively to programs such as pay for performance and prior authorization for medication or procedure orders. Recent emphasis on quality reporting and "meaningful use" [14] under the American Recovery and Reinvestment Act of 2009 (ARRA) gives further impetus to CDS, since CDS can be used to proactively improve performance on the very tasks being measured as a basis for stimulus fund incentives or subsequent reductions in reimbursements.

Nonetheless, successful approaches to CDS have not been broadly disseminated and adopted for a variety of reasons [1], including competitiveness, incompatibility of platforms, lack of standards, and under-utilization of electronic health records (EHRs) and computer-based provider order entry (CPOE) - major platforms in which to integrate CDS. But even for those with access to CDS, enormous, obstacles exist, including the lack of (1) sources of high quality medical knowledge in executable form, and (2) infrastructure and processes for managing and updating such knowledge and integrating it into applications. Addressing these needs is very expensive, if not prohibitive, for individual organizations, even large ones. In addition, the need for knowledge management capabilities has not yet been widely recognized except within a few of the larger medical centers that have been in the vanguard of CDS adoption. Further, standards for CDS representation and integration into applications are as yet incomplete.

In 2006 the U.S. Office of the National Coordinator for Health Information Technology (ONC) and the Agency for Healthcare Research and Quality (AHRQ) commissioned a project by the American Medical Informatics Association (AMIA) and produced The Roadmap for National Action on Clinical Decision Support, which proposed a coordinated approach to address the above impediments [15]. The report recommended a series of activities to improve CDS development, implementation and use throughout the United States to help enable improvements in health, and the quality, safety and efficiency of healthcare delivery.

There had been little evidence to date that health care organizations, public and private, were willing to share knowledge that they believe gives them a marketplace advantage. Nevertheless this had been done in other fields, even fiercely competitive ones like genomics research, e.g., in terms of contributions to GenBank [16], the Genome Knowledgebase [17], the DAVID Knowledgebase [18], Reactome [19], and other molecular and genetic data and knowledge bases. To the extent that it has occurred at all, the primary sharing of clinical knowledge in executable form has generally been limited to the activities of knowledge content providers, EHR vendors, or user groups of a particular vendor. Other ventures have been less than successful. As an example, the Arden Syntax was developed in the early 1990s, and was adopted as an ASTM standard in $1991[20,21]$ and subsequently moved to Health Level Seven (HL7) in 1998; but a website for sharing of Arden Syntax Medical Logic Modules (MLMs) developed at Columbia University in the mid-1990s did not get regularly updated, and is no longer available. Arden Syntax has considerable use, but with many proprietary implementations, effectively limiting the extent to which sharing occurs. A consortium called the Institute for Medical Knowledge Implementation was formed in the early 2000s, and included health information system vendors, academic medical centers, and professional societies as members, with the goal of creating shared medical knowledge modules. The group foundered when no entity was willing to contribute content. (See [22] for brief mention, although IMKI website is no longer available.)

Further, biomedical knowledge and technology development continue to advance relentlessly, making it impossible for practitioners to keep up with current knowledge, or for organizations to keep pace by integrating the new knowledge into their own practices. Promising sources of generally available knowledge such as AHRQsupported National Guideline Clearinghouse [23] and the Evidence-based Practice Center reports [24], and the Cochrane Collaboration Library [25] seek to establish and update authoritative collections of reports identifying best health care practices.

A current AHRQ program initiated as a part of the American Recovery and Reinvestment Act (ARRA) is the promulgation of Comparative Effectiveness Research (CER) [26], which promises to further enhance such efforts. There are clearly many policy issues involved, such as how to assemble and organize disparate studies, whether a coordinating center should exist, what its role in guiding clinical decisions or influencing health care spending should be, and how it should be governed to ensure appropriate representation of stakeholders [27]. Assuming that a politically acceptable approach for these difficult issues is found, these knowledge resources would still not be made available in unambiguously executable (or even nearexecutable) form.

The technical issues and impediments involved and possible approaches to bridging the gap between such reports and recommendations and the development of ready-toimplement CDS are elaborated in the AMIA Roadmap callto-action [15] and in the book by Greenes [1]. One attractive possibility that was highlighted in the call to action is the idea of developing a Web-accessible repository of high quality medical knowledge, in an unambiguous form that can be used as a basis for implementing CDS, and that would be available to all institutions and users. This would avoid the need for each organization to duplicate the effort of creating and maintaining such a repository. Responsibility for managing this communal repository would rest in an authoritative body that would determine knowledge to be 
included, formalize its representation, index it for retrieval, and keep it updated. Projects initiated over the past two years have begun to tackle this idea.

\section{A.2. The Morningside Initiative}

The Morningside Initiative began when a small group of prominent health care organizations came together for a working meeting at the Morningside Inn, Frederick, MD, August 28-30, 2007, under the sponsorship of the Telemedicine and Advanced Technologies Research Center (TATRC) of the US Army Medical Research and Materiel Command. The purpose was to develop a long-term plan intended to address the challenges by means of a deliberate, gradual process, initially involving selected participants for a limited set of tasks, and then expanding in scope and scale as workable approaches are developed. Participants included representatives of the Department of Defense (DoD) TriCare Management/Health Affairs (TMA/HA), the Veterans' Health Administration (VHA), Partners Healthcare System (Partners), Kaiser Permanente (Kaiser), Henry Ford Health System (HFHS), Arizona State University (ASU), the AMIA, and TATRC.

The Morningside Initiative is the result of that August 2007 initial working meeting, and was the unanimous recommendation of participants. It called for a collaboration to create a repository of shared knowledge for CDS to meet the needs of and be made available to diverse healthcare providers and organizations. Subsequent to the initial meeting, Intermountain Healthcare (Intermountain) joined the other participants to form the Morningside Initiative.

\section{A.3. Morningside Initiative Vision}

The Morningside Initiative is dedicated to
developing a collaborative Web-based
resource that supports the sharing of evidence-
based medical knowledge in executable form
for clinical decision support, in order to
improve the health of the community and the
quality of health care.

To achieve this vision, the collaboration is intended as a prototype of a larger future knowledge-sharing organization, aiming at developing and refining (1) an organizational framework, (2) a technical approach, and (3) content acquisition and management processes that are functional and can be scaled up to include a broader range of participants as well as an expansion of capabilities and content scope. The organizers of the Morningside Initiative concluded that the challenges of knowledge sharing (access to high quality content, reusability, tools for knowledge management, and an organizational framework to facilitate knowledge exchange) would best be addressed by bringing together its small number of highly visible and respected health care organizations in the public and private sector that could lead by example. They would develop and test a framework for knowledge sharing that could be extended to a national-level effort if successful. The rationale for this approach was based on three primary considerations:

a) Working out the organizational and logistical issues of knowledge sharing could be best done first with a small number of participants, so that the approaches can be refined through that experience, before undertaking a large-scale initiative involving many more participants.

b) Reluctance to share clinical knowledge by institutions could be overcome if high-profile participants have already committed to doing so and seeded the effort with a corpus of useful knowledge content.

c) Tools and methods for knowledge management, for creating, representing, and updating the content, and for facilitating adaptation and reuse in other sites should be developed and piloted with this small group.

The general framework for the Morningside Initiative is shown in Fig. (1). Current efforts are to capture best available knowledge already implemented as CDS in the systems of participant organizations; represent it in a more sharable, less setting-dependent format; drive toward standards-based models for representation; and develop and test approaches for secondary reuse. The key test of reuse is the adoption of this knowledge in settings different from those from which the CDS had originally been implemented.

In the future, it is anticipated that external knowledge will continually arise from authoritative studies, and that a process will be established to regularly incorporate this knowledge into the shared knowledge base. This is discussed further in the section on Future Directions.

The rationale for the effort has included the following potential benefits:

- In concert with the nationwide efforts to achieve widespread adoption of interoperable health IT, a collaborative approach based on standards and interoperability will help attain greater proliferation of CDS than any one entity could achieve alone.

- The approach will broaden the potential use and application of CDS at the point of care in order to impact health care decisions throughout the health care system.

- It will advance and foster more widespread accessibility of CDS content and interventions.

- $\quad$ By leveraging lessons, tactics and approaches used by others, buy-in across local, regional and national entities will be increased.

- A collective effort will address CDS topics of prime concern and importance to the national health care system in terms of cost, quality, and access to care.

- A national-level resource will facilitate adoption and more effective use of CDS within organizations that might otherwise not have the resources to pursue CDS.

- Availability of the resource will remove or lessen barriers and challenges (in terms of technical, cost, and expertise deficiencies) and thus enhance the likelihood of adoption and proliferation of CDS.

- Professional benefits will accrue to individual investigators for participation (e.g., papers, grant writing, research, and leadership opportunities). 


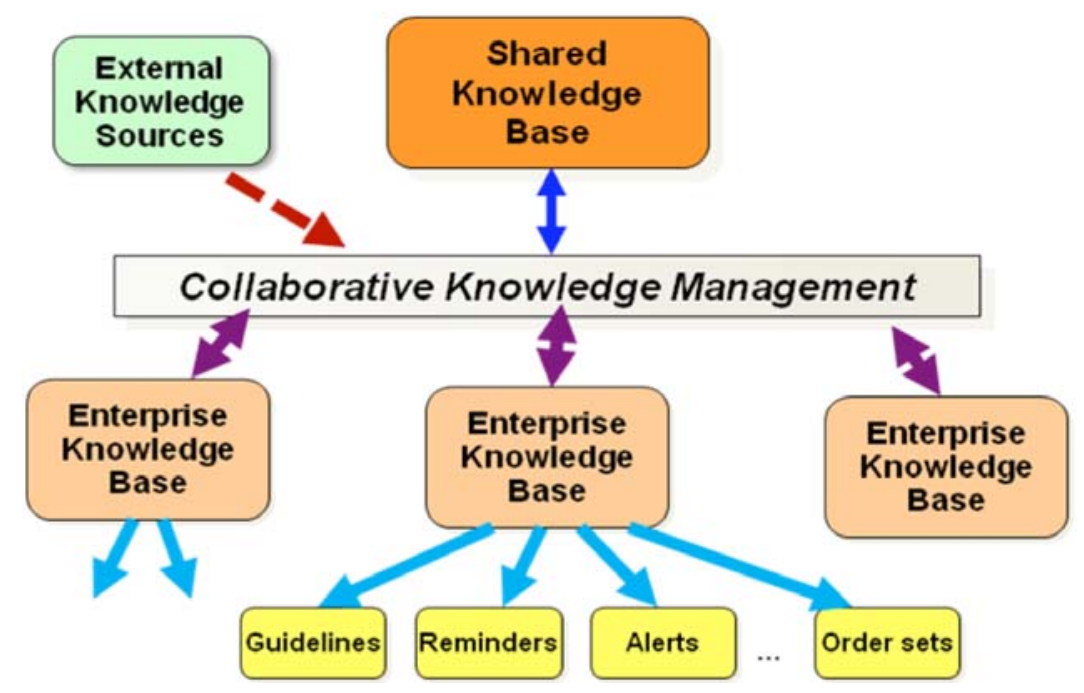

Fig. (1). General framework for the Morningside Initiative.

- The collaborative mechanism will provide participating entities with a level of external validation of their CDS work regarding content quality and development approach.

A recognized requirement for a future collaborative knowledge sharing organization is a business model based on sustainable long-term mechanisms of support, yet to be defined, which might be expected to include a combination of organizational subsidies or membership dues, governmental and payer funding, and possibly other mechanisms. For the short term, limited support has been provided through TATRC, and additional sources of support are being sought. Most of the activities described in this report have been carried out through the in-kind contributions of time and effort by the participants and their organizations.

\section{A.4. Morningside Initiative Scope and Relationship to Other Efforts}

The initiative described here does not duplicate the work of Evidence-based Practice Centers, the Cochrane Collaboration, Comparative Effectiveness Research, or other efforts to determine best practices and to develop guidelines based on meta-analysis and evidence-based medicine. An operational knowledge repository would, however, regularly draw upon the work of these organizations to operationalize their findings. For the near term, the Morningside Initiative takes as its starting point the "operationalized" rules-based knowledge that health care provider organizations have already determined to be useful and have implemented in various applications in their systems, usually in the form of decision rules for alerts, reminders, or medication prescribing recommendations, or as order sets. Typically these CDS modules have been drawn from guidelines, authoritative reviews or other evidence-based medicine sources, but they have been made unambiguous and computable - a process which sounds straightforward but is definitely not. Further, typically these modules are not represented in a language that can be interpreted by other systems or applications, even within the same organization. So a major focus is to develop a common shared representation for such knowledge.
A subsequent phase of the Morningside Initiative is anticipated, which includes a national-scale effort to maintain and update a shared repository of executable knowledge. This would include a regular process to review and incorporate new knowledge coming from such authoritative sources as above. However, that is not the initial focus of the effort.

In 2008, at about the same time as the Morningside Initiative was being formed, AHRQ issued contracts for two projects, the Guidelines into Decision Support (GLIDES) project and the Clinical Decision Support Consortium (CDSC). Like the Morningside Initiative, both GLIDES and CDSC are concerned with the lifecycle of transforming knowledge from narrative form to implementation. GLIDES is particularly focused on narrative guidelines and using the Guideline Element Model (GEM) and GEM Cutter tools [28] to implement narrative guidelines in testbed settings. The CDSC aims to develop cooperative approaches to share and manage CDS knowledge [29] The CDSC, which includes health care organizations and vendors, is using a top-down approach to develop knowledge management models, representations, and lifecycle processes, based on the internal knowledge management portal development at Partners Healthcare, and a proprietary content management platform (Documentum, EMC Corp., Hopkinton, MA).

The Morningside Initiative, by contrast is committed to adopting an open, collaborative process for knowledge management in order to create a shared repository of content for CDS that would be as close to implementable form as possible. Further, a goal of this group is to follow best available models for knowledge representation and to help drive the standards development process where appropriate standards are lacking.

The Morningside Initiative is working in collaboration with the Congressionally-funded Knowledge Management Repository (KMR) project, directed by CAPT Emory Fry, MD, at the Naval Health Research Center, San Diego, CA (see: [30]). The KMR project is aimed at developing an open-source platform for CDS for the Federal NHINConnect implementation. Thanks to this collaboration and a cooperative research and development agreement (CRADA), 
this NHIN-Connect hardware/software stack is installed at the ASU Clinical Application, Research, and Education Interoperability Testbed (CARE-IT) Laboratory [31], and is available for Morningside Initiative work. CARE-IT is a collaboration between academic, government and industrial partners established to promote research into open-source, interoperable and standards-based solutions for health care information technology (HIT) and to provide an innovative infrastructure for the education of health care and technology professionals. We use this resource as the technical framework for hosting the CDS knowledge repository, delivering and ensuring interoperability of the components of this research - both the content repository, tools, and resources needed for their support - and providing a collaboration environment for participants.

Another collaboration that has evolved is with the Structuring Care Recommendations for Clinical Decision Support (SCRCDS) project. This project, originally called the Hardened Rules Project, is a contract funded by AHRQ to Thompson Reuters, Inc., with subcontracts to Morningside Initiative participants at ASU and Intermountain Health, among others (see: [32]). A primary goal of that project is to develop a formulation for the United States Preventive Services Task Force (USPSTF) A and B-level prevention and screening recommendations (45 in all), that is unambiguous and sufficiently detailed to facilitate broad uptake and use by EHR implementers and vendors, as well as a small subset of rules based on Meaningful Use quality measures. This involves producing the rules in terms of welldefined data elements and logical constructs, but not incorporating setting-specific factors such as triggering conditions, workflow, and action details that limit their portability. Morningside Initiative participants are seeking to develop formal approaches to extending the output of the SCRCDS project to incorporate a systematic process for adding in the site-specific features, based on requirements of specific sites.

More recently, in 2010, ONC issued grants to institutions for four Strategic Health Advanced Research Projects (SHARP), One, known as SHARP-C, focused on Decision Making and Cognition, was obtained by the University of Texas Health Science Center at Houston to create a National Center for Cognitive Informatics and Decision Making in Healthcare [33]. A subset of the Morningside Initiative team involving ASU, Intermountain Healthcare, the VA Palo Alto Healthcare System, and the Naval Health Research Center is responsible for one of the projects, that of developing an "implementers' workbench". The goal of that effort is to create a resource for enabling CDS rules that capture evidence-based best practices but are not site-specific to be encoded with the triggering, data source, interaction, notification, and other workflow and setting-specific features necessary to be incorporated effectively into a specific site.

The Morningside Initiative's lifecycle model for knowledge acquisition, refinement, and deployment (see Section B) involves 4 stages of evolution of knowledge content that is the basis for the implementers' workbench. Fig. (2) shows the relationship of the Morningside Initiative to the foci of the KMR, SCRCDS, and SHARP-C projects. As can be seen, this evolution has enabled the Morningside Initaitive to become a kind of coordinating entity through which participants in other related projects are able to align their efforts.

\section{A.5. The Importance of Sharing, Standards, and Open Systems}

The ultimate purpose of the Morningside Initiative is to establish a continually updated and widely accessible national-level repository of authoritative knowledge in executable form. The details of the knowledge are to be fully transparent. That participation will be available to all is a guiding principle.

Since standards are still immature, the goal of being able to provide knowledge in standard form is not fully attainable. Nonetheless, the Morningside Initiative intends to represent knowledge in unambiguous form by using (or developing as needed) conventions for representation that are considered by the team to be the best available, and which are fully documented. The team also works with standards development organizations to help accelerate the adoption of standards that are most urgently needed. It is expected that as this effort gains traction it may provide an important use case for such standards development, to help drive the direction of the process.

A principle of the approach is that knowledge bases are managed using open source tools and non-proprietary data formats wherever possible. This avoids licensing fees or vendor dependence, or the delays in waiting for vendors to support particular needed features. Open platforms, e.g., the J2EE framework, have demonstrated scalability and performance, and there are growing numbers of open-source software products available for various needs.

\section{B. METHODS}

The Morningside Initiative takes a lifecycle approach to establishing the basis for sharing executable CDS knowledge. We envision that a starting point might be a best practice recommendation created through an evidence-based medicine (EBM) development process. The lifecycle envisions four stages of refinement

1. Creation of a semi-structured representation of the recommendation in human-readable form. This involves Identification of the individual components of the recommendation, analogous to the approach used by GEM and inserting them into a template or form.

2. Formalization of the data elements and logic of the recommendation. This addresses the core medical knowledge but does not contain any references to setting-specific factors like triggering conditions, workflow, application environment, or methods of responding to or processing action recommendations.

3. Establishment of a process for generating settingspecific versions of the recommendation. This involves creating an ontology of setting-specific factors, and an ability to create a meta-level representation of the recommendation conforming to a particular set of setting-specific factors.

4. Generation of an executable version of the knowledge suitable for incorporation into a host environment. This involves translating the meta- 


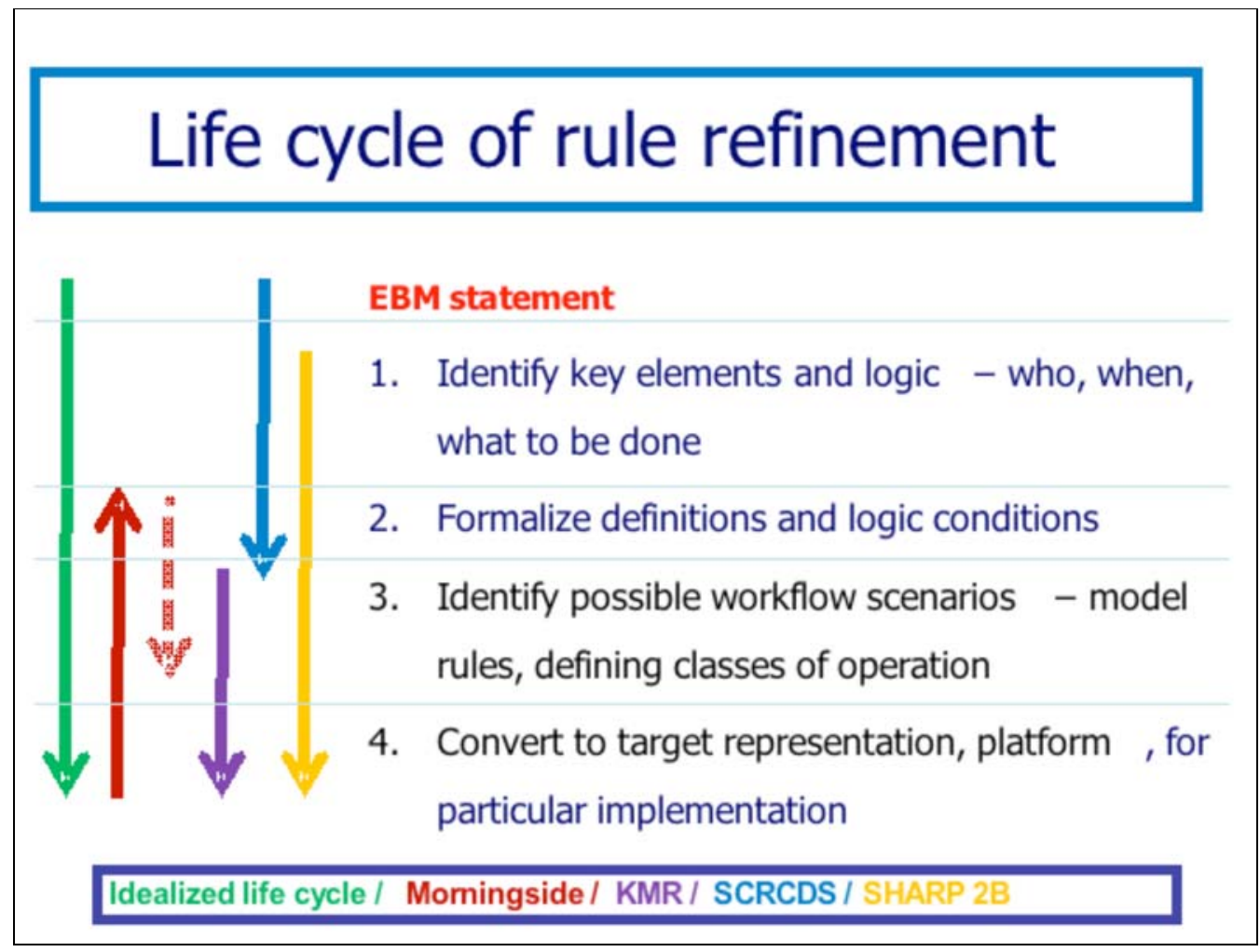

Fig. (2). Life Cycle of Rules Knowledge Refinement. Note that stage 3 is typically skipped by implementers, such as those deploying Arden MLMs. The Morningside Initiative seeks to make stage 3 explicit, as a means of capturing the experience of successful implementations and also as a means to formalize the range of setting-specific options needed to successfully embed CDS into operational settings. The Morningside Initiative is seeking to do this using open source tools and methods and a collaborative process wherever possible. Relationships to three concurrent projects involving Morningside Initiative participants, the DoD-based Knowledge Management Repository (KMR) project, the AHRQ-funded Structuring Clinical Recommendations for Clinical Decision Support (SCRCDS) project, and the ONCfunded SHARP-C subproject 2B are shown. Two AHRQ-funded initiatives, the Guidelines into Decision Support (GLIDES) and the Clinical Decision Support Consortium (CDSC), have similar goals of supporting the lifecycle shown in this figure, but are using different approaches. GLIDES is using the Guideline Element Markup (GEM) tools to refine guidelines into decision support implementation. CDSC is developing knowledge authoring, sharing, and execution tools and processes based on a formal model of intermediate knowledge artifacts.

level representation into the delivery form used by a host environment, e.g., in Arden Syntax, in a business rules language, or as SOA modules.

As shown in Fig. (2), the Morningside Initiative is concentrating primarily on stages 2 and 3 of the lifecycle process. Most existing knowledge in executable form is already at stage 3 or 4 , limiting its portability. Authoritative EBM guidelines and recommendations are typically in narrative form or at best at stage 1. For the Morningside Initiative, a natural starting point initially has been the operational knowledge at stage 4 in participating organization sites, which by analysis is transformed into stage 2 and stage 3 representations. The KMR project seeks to go from stage 2 to stage 4 in the form of SOA-based CDS modules, so the collaboration with KMR is focused on that use case as one target for its work. The SCRCDS project is focused on transforming USPS TF recommendations in the EBM narrative format stage into stage 1 and then stage 2 form, with guidance for transforming to stage 3 . The Morningside Initiative participants are treating this as another use case to determine what is necessary to transform the knowledge into delivery formats of use in participants' organizational environments. The SHARP-C subproject of the Morningside Initiative participants (SHARP-C project
2B) is aimed at facilitating the transformation across all stages through an implementers workbench, but with particular emphasis on stage 3 and the modeling of settingspecific factors to support that.

Besides the technical/infrastructure approach, Morningside Initiative goals include addressing the organization and content expertise for facilitating knowledge sharing. The Morningside Initiative is governed by a Steering/Organization Committee overseeing two other Committees functioning as Working Groups. The committee responsibilities are as follows:

- Steering/Organization Committee $\quad-$ Initially composed of representatives of all founding organizations, the Steering Committee is expected to evolve into elected membership based on constituencies, such as professional specialty societies, health care delivery organizations, knowledge content provider entities (commercial and otherwise), and other stakeholders to be defined. This committee oversees the development and refinement of the bylaws operating procedures, conducts governance business, and oversees the evaluation and sustainability aspects of the Initiative. 
- Content Committee - This Committee is responsible for specifying content focus priorities, acquisition, review, and rating procedures, and overseeing the content update to the shared knowledge repository (KR). It will also develop processes for annotating and resolving potentially contradictory knowledge resources.

- Infrastructure and Technology Committee - This committee is responsible for functional requirements, tools specification, development and testing, and deployment/technical operations. It is also the primary interface for engagement with the standards development process through participation in HL7, OASIS, and other SDOs.

The Morningside Initiative has adopted a management strategy that we believe to be truly inclusive and collaborative. The above committees, when they identify a task to be performed, designate a committee liaison person (either on the committee or approved by the committee membership) as the one responsible for overseeing that task. Together with the committees, the scope of the project, resource requirements, priority, and timeline are determined, and if approved by the committee, the resources are allocated from the technical team. The coordination of requests is overseen by a Project Management Team of the Steering/Organization Committee.

Specific activities include the following:

\section{B.1. Establish Open Source, Standards-Based, Shared Knowledge Repository}

The functional requirements for delivery define needs for the CDS, the process for selecting and rating the CDS for inclusion in the KR, the tools and methods for representing it, and approaches for contextualizing it for use. Ideally, we further impose that the content and tools be standardsbased and open source, wherever possible, and that the processes involved in implementing the life cycle be that of an active, engaged, viral open source community of knowledgeable developers and users. A draft set of functional requirements (some of which are included in Table 1) forms a basis for our approach and is being further refined.

As noted above, the initial focus in the Morningside Initiative has been on CDS content which is already implemented in existing participant sites and which has been shown to be effective. The aim is to learn from examining that content what the unique features of each implementation are, to compare knowledge aimed at similar purposes, and to forge a common representation. Knowledge content foci are determined by the organization/steering committee, and are initially primarily aimed at high priority clinical conditions, notably involving chronic disease. We have further restricted our activities thus far to examination of diabetes rules-based knowledge, e.g., HbAlc testing, Metformin prescribing, eye and foot examination reminders, and operational definitions of chronic diseases.

Once having created a sharable representation, abstracting context-specific and site-specific aspects, the goal is to re-contextualize the content to enable it to be incorporated into another site with distinct EHR platform, organization, and workflow requirements. This analysis of content has to date been very instructive, and has identified the need to separate the core medical knowledge (the stage 2 representation, as per Fig. 2) from the business or workflow logic and to characterize a variety of annotation meta-tags (see Table 2) that relate to things like how the CDS is triggered, how and where it interacts with applications and users during evaluation, and how the results are communicated or inserted into the workflow (the stage 3 representation).

We have also shown that many apparently different rules are for the same medical purpose, once one does the above analysis. This is exemplified in Fig. (3), which compares knowledge content gathered from Morningside Initiative participant implementations dealing with various diabetesrelated rules. For example, a variety of different rules are seen all dealing with how to deliver a reminder that HgbAlc testing should occur every 6 months. It is clear that the differences in appearance are all due to the triggering, presentation, and other business logic considerations. Thus a major task of this initiative is to formalize the content acquisition process in order to understand how to represent these layers.

\section{B.2. Develop Robust Content Knowledge Management Practices and Technical Resources and Procedures, Methodologies, and Representations of Knowledge}

The key innovation in the technical approach is to put together a variety of emerging open source capabilities and to engage the community in an active collaborative mode in its development, testing, and refinement.

Functional requirements development. The evolving set of functional requirements for the Morningside Initiative has been prepared together with those being developed for the KMR project. The latter are aimed more at the end-user delivery phase of CDS, but we believe that the requirements for the knowledge model and representations cannot be divorced from the functional requirements for the delivery of CDS, and thus we are evolving these together. As noted above, see Table $\mathbf{1}$ for draft Morningside Initiative requirements.

Using tested content as a starting point, we can address two of the key needs surrounding the transfer of CDS between sites: (1) detailed documentation of the functional requirements for each of the components found in an environment that supports CDS, and (2) a collection of use cases from which we can catalog characteristics of the clinical workflow and the clinical environments necessary for the successful delivery of CDS.

This functional requirements draft document indicates anticipated capabilities of a knowledge repository, of a knowledge authoring and maintenance system, of a service to support the sharing of this knowledge, and finally, of a typical system for executing logic imported through this service. Since these functional requirements are derived from the experience of multiple institutions, no specific language or implementation is described. The goal of the requirements document is to be complete, to the extent that a system developer might choose to implement a subset of the 
Table 1. Condensed Functional Requirements for a Knowledge Repository. Requirement Categories and Examples of Functional Requirements are Included. The Requirements Seek to Capture Elements of the Knowledge Model, the Knowledge Authoring Environment, the Knowledge Sharing Environment, and the Repository itself. An As-Yet-Unrealized Goal is to Specify Functional Requirements for a Knowledge Execution Component of this Environment

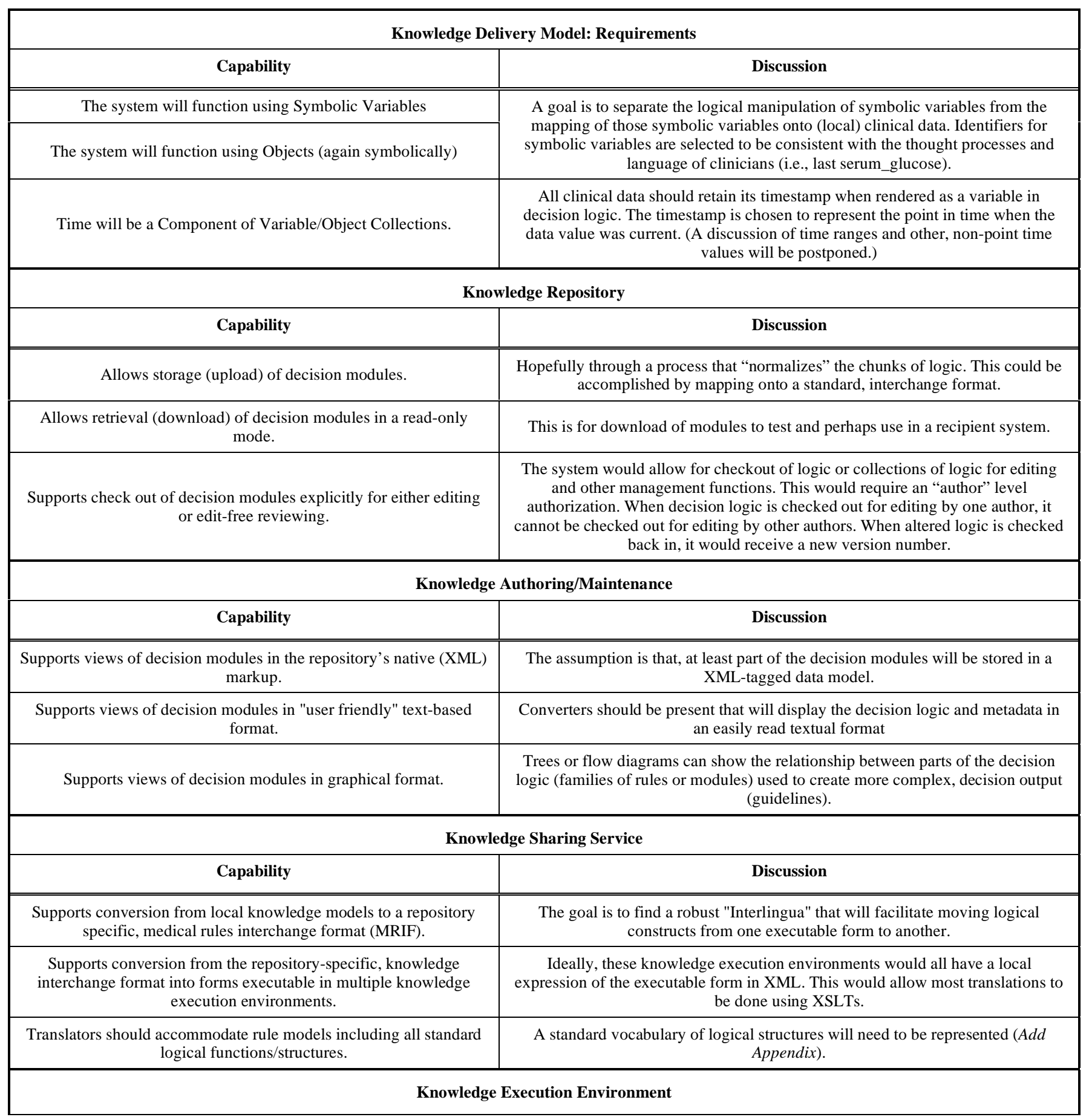

described functions and have a usable system. However, this detailed level of specification is appropriate for a knowledge repository, since such a repository would be expected to manage logic from sites that have implemented differing subsets of the defined functionality.

The second component of this analysis involves an aspect of CDS that clearly requires more study. Medical decision modules can be integrated into a clinical workflow in a variety of different ways. Similar logic could support alerts, observations displayed in clinical worksheets, suggested orders in a CPOE system, and a number of other potential delivery mechanisms. We believe that this attention to clinical work processes is essential for successful implementation of CDS. A knowledge repository that fails to document the approach, timing, and context of the delivery of a CDS intervention would leave a site that wishes to 
Table 2. Examples of Metadata for Indexing Content in a Knowledge Repository. The Collection is Derived from Metadata Described in a Number of Categorization Schemes for Computable Medical Knowledge

\begin{tabular}{|c|c|c|}
\hline Metadata Title & Metadata Description & Orig. Metadata Source \\
\hline Resource Title & Title of module of clinical logic. & $\begin{array}{ll}\text { - } & \text { In Arden } \\
\text { - } & \text { In Morningside Template }\end{array}$ \\
\hline File Identifier (URI) & Uniform Resource Locator(for storage); replaces filename (Arden) & $\begin{array}{ll}\text { - } & \text { In Arden } \\
\text { - } & \text { In Morningside Template }\end{array}$ \\
\hline UNID & Unique identifier for indexing through a terminology service for instance. & - In Morningside Template \\
\hline $\begin{array}{l}\text { Version and } \\
\text { Branch ID }\end{array}$ & $\begin{array}{l}\text { A number indicating the version. In the context of a source repository, this might } \\
\text { capture new branches as versioning generates alternate directions in the logic. }\end{array}$ & $\begin{array}{ll}\text { - } & \text { In Arden } \\
\text { - } & \text { In Morningside Template }\end{array}$ \\
\hline $\begin{array}{l}\text { Submission/Revision Dates/ } \\
\text { Branching dates }\end{array}$ & The date associated with submission of this version. & $\begin{array}{ll}\text { - } & \text { In Arden } \\
\text { - } & \text { In Morningside Template }\end{array}$ \\
\hline Purpose & $\begin{array}{l}\text { Statement of the goal of the logic (free text). May also provide tag references to a } \\
\text { controlled taxonomy of purposes. }\end{array}$ & $\begin{array}{ll}\text { - } & \text { In Arden } \\
\text { - } & \text { In Morningside Template }\end{array}$ \\
\hline
\end{tabular}

\begin{tabular}{|c|c|c|c|c|c|c|c|c|c|c|c|c|}
\hline \multirow{3}{*}{\multicolumn{2}{|c|}{ HGBA1C_ASSESSMENT }} & \multicolumn{4}{|c|}{ PHS } & \multicolumn{4}{|c|}{ IH } & \multirow{3}{*}{\multicolumn{2}{|c|}{ VA }} & \multirow{3}{*}{ KP } \\
\hline & & \multirow{3}{*}{\begin{tabular}{|l|}
$\begin{array}{l}\text { Over } \\
\text { due }\end{array}$ \\
\end{tabular}} & \multirow{3}{*}{\begin{tabular}{|c|}
$\begin{array}{c}\text { Expir } \\
\text { ing }\end{array}$ \\
\end{tabular}} & \multicolumn{2}{|c|}{ High } & \multirow{3}{*}{15} & \multirow{3}{*}{16} & \multirow{3}{*}{17} & \multirow[b]{2}{*}{18} & & & \\
\hline & & & & 1 & 2 & & & & & & & \\
\hline \multirow{10}{*}{ Conditions } & Most recent $\mathrm{HgbA} 1 \mathrm{c}<($ or $<=) 9$ months old & & & & & & & & & & & $\mathrm{N}$ \\
\hline & Most recent HgbA1c $<$ (or $<=$ ) 11 months old & & & & & & & & & $\mathrm{N}$ & $\mathrm{N}$ & \\
\hline & Most recent $\mathrm{HgbA} 1 \mathrm{c}<$ (or $<=) 6$ months old & $\mathrm{N}$ & $\mathrm{Y}$ & & & & $\mathrm{N}$ & & & & & \\
\hline & Most recent $\mathrm{HgbA} 1 \mathrm{c}<($ or $<=) 5$ months old & & $\mathrm{N}$ & $\mathrm{Y}$ & & $\mathrm{N}$ & & & & & & \\
\hline & Most recent $\mathrm{HgbA} 1 \mathrm{c}<($ or $<=3$ months old & & & $\mathrm{N}$ & $\mathrm{Y}$ & & & $\mathrm{N}$ & $\mathrm{N}$ & & & \\
\hline & Most recent $\mathrm{HgbA1C}$ (in the last year) $>7 \%$ & & & $\mathrm{Y}$ & $\mathrm{Y}$ & & $\mathrm{N}$ & $\mathrm{Y}$ & & & & \\
\hline & Most recent $\mathrm{HgbA} 1 \mathrm{c}$ (in the last year) $>8 \%$ & & & & & & & $\mathrm{~N}$ & $\mathrm{Y}$ & & & \\
\hline & HgbA1c documented elsewhere & & & & & & & & & $\mathrm{N}$ & & \\
\hline & HgbA1c ordered within last 7 days & & & & & & & & & & & $\mathrm{N}$ \\
\hline & Patient refused HgbA1c testing within last $6 \mathrm{mo}$ & & & & & & & & & $\mathrm{N}$ & $\mathrm{Y}$ & \\
\hline \multirow{11}{*}{ Messages } & Patient is overdue for $\mathrm{HbA} 1 \mathrm{c}$ (rec: 96 months) & $\bar{Y}$ & & & & & & & & & & \\
\hline & Missing HgbA1c data (should be done on all Patients with Diabetes) & & & & & $\mathrm{Y}$ & & & & & & \\
\hline & All Patients with Diabetes should have a HgbA1c at least every 6 months & & & & & & $\mathrm{Y}$ & & & & & \\
\hline & Diabetics need at least annual Hemoglobin A1C testing. & & & & & & & & & & & $\mathrm{Y}$ \\
\hline & $\begin{array}{l}\text { Hemoglobin A1C required annually for all diabetic patients. Patients with levels }>7.0 \text { should be } \\
\text { considered for more frequent testing. }\end{array}$ & & & & & & & & & $\mathrm{Y}$ & & \\
\hline & Patient is almost due for $\mathrm{HbA1c}$ (rec: $q 6$ months) & & $\mathrm{Y}$ & & & & & & & & & \\
\hline & All Patients with Diabetes and $\mathrm{HgbA} 1 \mathrm{c}$ between 7 and 8 should have a $\mathrm{HgbA} 1 \mathrm{c}$ every 3 months & & & & & & & $\mathrm{Y}$ & & & & \\
\hline & All Patients with Diabetes and $\mathrm{HgbA} 1 \mathrm{c}>8$ should have a $\mathrm{HgbA} 1 \mathrm{c}$ every 3 months until under 8.0 & & & & & & & & $\mathrm{Y}$ & & & \\
\hline & Last $\mathrm{HbA} 1 \mathrm{c}$ is high, and patient is overdue for $\mathrm{HbA} 1 \mathrm{c}$ (rec: $q 3$ months) & & & $\mathrm{Y}$ & & & & & & & & \\
\hline & Last $\mathrm{HbA} 1 \mathrm{c}$ is done within 3 months, but high & & & & $\mathrm{Y}$ & & & & & & & \\
\hline & Patient refused Hemoglobin A1C testing. Ask again in 6 months. & & & & & & & & & & $\mathrm{Y}$ & \\
\hline \multirow{2}{*}{ LAB } & Order A1C test today & $\mathrm{Y}$ & $\mathrm{Y}$ & $\mathrm{Y}$ & & & & & & & & \\
\hline & Order A1C test in 3 months & & & $\mathrm{Y}$ & $\mathrm{Y}$ & & & & & & & \\
\hline
\end{tabular}

Fig. (3). Comparison of example rules from Partners Healthcare, Intermountain Healthcare, Veterans Administration, and Kaiser Permanente dealing with HgbA1c assessment. Columns indicate individual rules (or disjunctive clauses of rules) by institution. The conditions potentially evaluated are enumerated in the blue section, with those included in a particular rule indicated by the value needed for satisfaction (N or $\mathrm{Y}$ ). The actions to be performed (sending of a message or ordering of a test) on satisfaction of the conditions for a rule are indicated in the red and yellow sections of that column by the presence of a Y.

import these rules with documentation inadequate to do so successfully.

As we proceed in this work, we anticipate evaluating CDS logic from a number of institutions. We will treat these as "use cases" from which we will document the characteristics of the work processes that are used in successful CDS implementations. We anticipate developing or adopting templates and terminologies appropriate to capturing this information. This documentation will make the character and implications of the chosen intervention clear to any manager of a decision support environment who attempts to implement logic imported from the KR.

Tools and architectural approaches based on KMR project. The KMR project has developed an NHIN- compatible set of interoperable components that the Morningside Initiative intends to build upon for its work. The KMR focus has been to demonstrate that CDS material can be retrieved from a shared repository and executed within both military and civilian health information systems. It seeks to create an open source infrastructure, based on the FHA NHIN-Connect open source release, for sharing domain knowledge and executing CDS. The uniqueness of this approach is that it will not only result in an open standards platform with standardized application program interfaces (APIs) and services, but it will also contribute to the growth of a collaborative academic community, dedicated to improving health care. To support ongoing, iterative improvement in functional and technical 
capabilities, the system is being designed to collect performance and usability metrics.

Additional tools, methods, and processes. As part of its ongoing work, the Morningside Initiative seeks to adapt from the above, obtain externally, or build other tools and processes to construct, maintain and use CDS knowledge in a KR.

Several aspects of the KR environment deserve mention. First, the long-term goal is to conveniently host knowledge for thousands of decision modules over long time periods. This implies that there must be good tools for searching for decision modules, versioning decision modules, importing and exporting decision modules, testing decision modules, etc. In addition, the repository needs to contain more than just the decision logic. We have begun to assemble a list of metadata (see Appendix B) that we anticipate will be necessary for the management of this decision logic as well as to support queries from users of this system searching for logic to import into their own clinical information systems.

Additional components of the KR will include several forms of knowledge documentation. Users searching for CDS logic to implement in their local medical environment will wish to review experience with specific collections of knowledge before they import and implement this logic. We anticipate providing for storage and retrieval of both unstructured and structured documentation. Pointers to appropriate articles and other documents will be provided, as would forms that allow a knowledge depositor to record measures of the success of the logic within the submitting institution. A user making a knowledge withdrawal would have access to this information to help predict the effectiveness of these CDS modules in his/her institution. We expect to use Web 2.0 rating and commenting methods for enriching the knowledge in the repository with user experience and observations.

Examples of open source tools and platforms that we intend to explore are (a) the NHIN-Connect tools, (b) the BioCore collaboration portal being fielded at ASU, built on a .NET framework and supporting workspaces for collaboration; (c) content management environments such as Alfresco (open-access derivative of Documentum); and (d) Drools, a rules engine system.

\section{B.3. Establish an Operational Public-Private Consortium}

The organization of the Morningside Initiative is subject to a set of procedures set forth in a Bylaws document. The Morningside Initiative is a public-private collaboration organized under a memorandum of understanding (MOU) signed by all participants. The Morningside Initiative and AMIA have agreed in principle for the Morningside Initiative to be affiliated under AMIA as an intermediate means to formalize the entity and carry out the business on behalf of its constituency. This was done as a way to maximize flexibility for long-term operation and sustainability as the entity expands to be a fully inclusive national-scale initiative. Other organizational and/or governance structures or approaches may be considered in the future.

Draft bylaws and the proposed AMIA affiliation agreement have been developed. Bylaws include language that addresses indemnification and intellectual property protection of contributors of knowledge content, as well as the Morningside Initiative itself. Participants sharing content will need to be indemnified by clinical care organizations accessing and implementing this content in their respective clinical decision support systems. Further, those sharing content will be responsible for respecting their own third-party content intellectual property agreements as well as protected from having their shared artifacts sold by other third parties.

The original intent in forming the Morningside Initiative was to limit the number of participants initially, until the organization, content, and technical approaches were sufficiently defined, before expanding. We have learned much in the past year about the nature of the collaboration process, however, and that it may be possible to actually move more quickly to a broader scale, by fully embracing an open source, open collaboration model. A key reason for this is that we have found that the interest in this activity is much broader than we had originally believed, given the general reluctance to sharing of CDS over the past decade. A large part of this is due to the focus of ONC, AMIA, and various leading health care organizations on CDS, and the new vibrancy of open source communities in production environments, notably in health care.

The formal adoption of the bylaws has been slowed by the recognition that government participants such as the VA and DOD could not be signers. The current status is that the team is operating without a formal agreement in place, because of the flurry of activities in relation to the KMR, SCRCDS, and SHARP-C projects described in Section A.4. Through these projects, we have, in the short term, been able to further the goals of the Morningside Initiative through alignment of our participation in those projects, carried out under their auspices, with Morningside Initiative coordination, so that operation without the Morningside Initiative's own bylaws in those contexts has not impeded progress. We expect that formal adoption of bylaws will be necessary once it becomes desirable to expand the scope of participants in a broader consortium.

Regarding knowledge content sharing, efforts has been focused to date on a subset of rules relating to chronic disease management, particularly diabetes, and all participants have contributed their content without restriction. Thus, given current focus on the modeling and tool development around a subset of content, the broader issues of knowledge content sharing have been sidestepped for now, even though the draft bylaws address them. Again, we expect that we will need to turn back to this once the scope of participation in the Morningside Initiative expands.

\section{B.4. Drive Standards Evolution and Adoption}

A long-term goal of the process described above is to create a resource that will become increasingly used and valued as more and more of the management and documentation of health care in the U.S. is carried out electronically. We are convinced that development of and adherence to standards are central to effective implementation of EHRs and CDS. We are committed to using existing standards in this knowledge repository and, where standards are insufficient or do not exist, we anticipate 
applying the lessons learned in the analysis and design of the repository to promote extension of standards or to create new standards as needed.

Two kinds of standards are particularly applicable for those who wish to share CDS knowledge. The first is the language in which this knowledge is expressed. In the realm of medicine, two overlappingHL7 standards exist. These are the Arden Syntax for medical logic modules [21] and the GELLO expression language [34]. However, neither of these has seen widespread adoption in the medical computing community, except in the case of Arden Syntax, within vendor-specific implementations. An interesting alternative to these standards is represented by the growing use of business rules engines and other, general-purpose decision tools in some clinical environments. It appears that a repository that supports a single, computable representation of CDS logic will not be adequate.

In this context, the Morningside Initiative has begun to explore the possibility of an XML-based Interlingua as a tool for translating among different logic representations. Examples of such tools include the W3C RuleML [35] and a version of the Arden Syntax expressed fully in XML [36]. One of the goals of this effort will be to evaluate a subset of the existing (medical and nonmedical) standards to determine if the development of a medical rules interchange format is possible. The key goal of such an interchange format would be to support translations of CDS logic between languages.

In this activity we anticipate leveraging the work of others. The Morningside Initiative and KMR collaborators have regular interactions with a variety of standards organizations and expect to promote the adoption or development of a medical rules interchange format if further analysis confirms the feasibility of this approach.

The second kind of standard that is essential to the sharing of CDS logic is a mechanism to readily adapt decision logic built using data of one institution for use with data queried from the clinical database of a second institution. The central challenge when importing medical logic into a new clinical setting is to bind this logic to local data models and terminologies. This problem is known generically as the "curly braces" problem, arguably a key reason for the limited dissemination of the Arden Syntax as a mechanism for exchanging medical knowledge.

The Morningside Initiative plans to approach this challenge through analysis of two evolving standards. One of these, the virtual medical record (vMR) offers the option of mapping a nonstandard data representation onto a simplified version of the HL7, version 3, data messaging standard. The other, a product of the Healthcare Services Specification Project (HSSP), standardizes CDS as a service. The mapping of local data to the symbolic forms used in the CDS logic occurs during the construction of the message used to invoke the service.

We believe that a key product of our future work will be examples, use cases, and functional requirements designed to support the implementation of decision logic, imported from the repository, in settings where data models and terminologies differ from those present in the originating site.

\section{B.5. Demonstrate Suitability and Value for Secondary Reuse}

We are exploring this concept in two ways. One is adaptation to local processes/workflows, and the other is adaptation to local data representations. In these activities, we seek to use standards wherever adequate. We intend to demonstrate the ability to technically interface the CDS into different platforms. One target is the DOD AHLTA system being explored through the KMR project. Another, to be done between KMR and the ASU CARE-IT lab, is the Resource and Patient Management System (RPMS) of the Indian Health Service. In both cases, the approaches to be pursued involve use of the vMR and the HSSP efforts to model decision support as a service.

\section{B.6. Evaluate and Disseminate Program Status and Progress}

Since our collaborative process is intended to be an open one, a key dissemination strategy will be through use of a web portal. We intend to promote its use through panels and presentations at national meetings and online announcements. Through instrumentation of the web portal and inclusion of Web 2.0 assessment tools which are part of the BioCore portal design, we will track, monitor and assess content growth/update, and assess usability of tools and processes. We will also monitor cost of participation of each site and of coordination/central Morningside Initiative activities; usage $\operatorname{logs}$ and internal adoption by sites from repository; and governance issues/conflicts/resolution, and will publish and disseminate our results.

\section{CURRENT STATUS AND FUTURE DIRECTIONS}

The Morningside Initiative supports the following Critical Path Tasks identified in the AMIA Roadmap: it creates a mechanism whereby an ongoing forum for dialogue, consensus, and action by CDS stakeholders can be achieved; promotes dissemination and application of best CDS implementation practices; demonstrates the feasibility, scalability, and value of a collaborative approach to CDS by having specific, standardized tools and best practices publicly available; and provides a forum to analyze and generalize lessons learned from the development of a knowledge repository and its effects in furthering CDS.

The above processes have been going on for approximately twenty-four months, and the functional requirements, metadata tags, submission templates, and a collection of diabetes rules knowledge from the participating sites have been analyzed. These serve as a basis for a current effort aimed at finalizing the selection of a software and hardware platform for subsequent build out and development of the repository and tools, and gearing up the knowledge acquisition, formalization, and management tasks.

We believe that an important future effort will be authoritative review of best available knowledge that is ready for widespread use, and to be proactive in acquiring this new knowledge, formalizing it, and integrating it into the repository to facilitate dissemination and adoption. The aforementioned AHRQ-funded SCRCDS project focusing on the USPS TF A and B recommendations is an example of this focus. The SHARP-C project further focuses on the methodology for adapting to setting-specific factors. Thus, 
as illustrated by the SCRCDS and SHARP-C projects, a future direction will be to link up with and work closely with knowledge formalization activities of these natures, so as to close the loop between identified best practice and implementation.

Having such mechanisms will ultimately lead to the need for a decision in terms of the role of a national shared repository. We believe that the original content from a variety of sources, having been normalized, should be available for inspection by all users. But this is of primary benefit to secondary adopters that have needs very similar to the contributors or the wherewithal to conduct review of multiple variations and do the necessary adaptations themselves. There is a larger community of potential adopters who lack such expertise. Also, there is continued new knowledge arising from clinical trials, EPC, Cochrane, and CER reports, which finds its way into guidelines that have not yet been reviewed for implementability or relationship to existing CDS. Such review is a laborintensive and expensive process. Reviewing evidence from clinical trial and other sources for application to clinical care also requires unique expertise not only in the clinical content domain, but also in the evaluation of study design.

Large healthcare organizations that engage in developing their own guidelines devote substantial resources to this process. Small healthcare institutions such as individual hospitals and small group practices rarely have the resources to develop their own sets of knowledge content. Even large organizations are generally not able to produce revisions to guidelines more often than every few years. Thus this task may be important for a future national CDS initiative to address.

\section{C.1. Sustainability}

Ultimately, a national-level initiative will have participants in both public (federal/non-federal) and private (commercial/non-commercial) sectors, and with potentially many kinds of stakeholders (providers, payers, professional societies, government agencies, standards organizations, knowledge providers, and health systems providers, to name a few). One model for sustainability would thus be long-term commitment for support by a Federal agency, much as AHRQ supports the EPCs. An alternative is to establish a means for such an initiative to obtain support from its constituency. One model is that used by membership organizations, such as AMIA and HL7. These have both institutional and individual membership categories, with benefits associated with each, but do not limit access of members to their products and services based on type of user. Rather, the fee model essentially offers "quantity discounts" based on numbers of members. Such organizations have boards of directors that govern them, and which are open to broad participation through democratic processes.

The Morningside Initiative is a first step in bringing together on a voluntary basis organizations that have taken a leadership role in developing, deploying, and demonstrating the value of CDS for health care, to pool their expertise for the purpose of jump-starting a national knowledge sharing activity. The road ahead is long, but we have already learned that much can be gained by traveling together.

\section{DISCLAIMER}

The KMR project is supported by Award Number: W81XWH-06-2-0074, administered through the U.S. Army Medical Research Acquisition Activity, 820 Chandler Street, Fort Detrick, MD 21702-5014.

The opinions of the authors do not necessarily state or reflect those of their respective employers, including the Department of Veterans Affairs or the Department of Defense of the United States Government, and shall not be used for advertising or product endorsement purposes.

\section{REFERENCES}

[1] Greenes RA, Ed. Clinical Decision Support: The Road Ahead. New York: Elsevier 2007.

[2] Poisal JA, Truffer C, Smith S, et al. Health spending projections through 2016: modest changes obscure part D's impact. Health Aff 2007; 26(2): W242-53.

[3] National Coalition on Health Care. Health care Facts: Costs. 2009; [Retrieved: 11 November 2010]. Available from: http://nchc.org/fa cts-resources/ fact-sheet-cost.

[4] California Health Care Foundation. Health Care Costs 101. 2005; [Retrieved: 11 November, 2010]. Available from: http://www.chcf. org/

[5] Kohn L, Corrigan J, Donaldson M. Eds. and Committee on Quality of Health Care in America, Institute of Medicine. To Err Is Human: Building a Safer Health System. Washington, D.C., National Academies Press 1999.

[6] Institute of Medicine. Crossing the quality chasm: a new health system for the 21 st century. Institute of Medicine. Washington D.C., National Academy Press 2001.

[7] McGlynn EA, Asch SM, Adams J. The quality of health care delivered to adults in the United States. N Engl J Med 2003; 348(26): 2635-45.

[8] Mangione-Smith R, DeCristofaro AH, Setodji CM, et al. The quality of ambulatory care delivered to children in the United States. N Engl J Med 2007; 357(15): 1515-23.

[9] Balas EA, Boren SA. Managing clinical knowledge for health care improvement. In: Van Bemmel J, McCray AT, Eds. Yearbook of Medical Informatics. Stuttgart, Germany: Schattauer Verlagsgesellschaft mbH 2000; pp. 65-70.

[10] Khoury MJ, Gwinn M, Yoon PW, Dowling N, Moore CA. Bradley L. The continuum of translation research in genomic medicine: how can we accelerate the appropriate integration of human genome discoveries into health care and disease prevention? Genet Med 2007; 9(10): 665-74.

[11] McKinsey Global Institute. Accounting for the cost of U.S. health care: A new look at why Americans spend more. 2008; [Retrieved: 11 November 2010 from http://www.mckinsey.com/mgi/publicat ions/ US_healthcare/

[12] Halamka J, Overhage JM, Ricciardi L, Rishel W, Shirky C, Diamond C. Exchanging health information: local distribution, national coordination. Health Aff (Millwood) 2005; 24(5): 1170-9.

[13] Marchibroda JM. The impact of health information technology on collaborative chronic care management. J Manag Care Pharm 2008; 14(2 Suppl): S3-11.

[14] American Health Information Management Association. Meaningful Use-Provider Requirements. AHIMA Meaningful Use White Paper Series. Paper no. 2 2010; (Jan 20); 4 pgs, [Retrieved: November 11, 2010]. Available from: http://library.ahima.org/ xpedio/groups/public/documents/ahima/bok1_046481.hcsp?dDocN ame=bok1_046481.

[15] Osheroff J, Teich J, Middleton B, Steen E, Wright A, Detmer D. A Roadmap for National Action on Clinical Decision Support. Report. Bethesda, MD, American Medical Informatics Association, 2006.

[16] Benson DA, Karsch-Mizrachi I, Lipman DJ, Ostell J, Sayers EW. GenBank. Nucleic Acids Res 2010; 38(Database issue): D46-51.

[17] Joshi-Tope G, Vastrik I, Gopinath GR, et al. The Genome Knowledgebase: a resource for biologists and bioinformaticists. Cold Spring Harb Symp Quant Biol 2003; 68: 237-43.

[18] Sherman BT, Huang da W, Tan Q, et al. DAVID Knowledgebase: a gene-centered database integrating heterogeneous gene annotat- 
ion resources to facilitate high-throughput gene functional analysis. BMC Bioinformatics 2007; 8: 426.

[19] Vastrik I, D'Eustachio P, Schmidt E, et al. Reactome: a knowledge base of biologic pathways and processes. Genome Biol 2007; 8(3): R39.

[20] Hripcsak G, Wigertz OB, Kahn MG, Clayton PD, Pryor TA. ASTM E31.15 on health knowledge representation: the Arden Syntax. Stud Health Technol Inform 1993; 6:105-12.

[21] Pryor TA, Hripcsak G. The Arden syntax for medical logic modules. Int J Clin Monit Comput 1993; 10(4): 215-24.

[22] PRNewswire reference to the Institute for Medical Knowledge Implementation. 2003, [Retrieved: 11 November, 2010]. Available from: http://www.prnewswire.com/cgi-bin/stories.pl?ACCT=104\& STOR Y=/www/story/02-11-2003/0001889281\&EDATE=

[23] Agency for Healthcare Research and Quality. National Guidelines Clearinghouse. [Accessed: 11 November, 2010]. Available from: http://www.guidelines.gov, 2010;

[24] Agency for Healthcare Research and Quality. Evidence-based Practice Centers. [Accessed: 11 November, 2010]. Available from: http://www.ahrq.gov/clinic/epc/, 2010;

[25] The Cochrane Collaboration. The Cochrane Library 2010; [Accessed: 11 November, 2010]. Available from: http://www. thecochranelibrary.com/view/0/index.html

[26] Agency for Healthcare Research and Quality Fact Sheets on Recovery Act Investments in Comparative Effectiveness Research. 2010; [Accessed: 11 November, 2010]. Available from: http://ww w.ahrq.gov/fund/cerfactsheets/,;

[27] Wilensky GR. The policies and politics of creating a comparative clinical effectiveness research center. Health Aff (Millwood) 2009; 28(4): 719-29.

[28] Shiffman R. GLIDES Project: Guidelines into Decision Support. Slide presentation from the AHRQ 2009 Annual Conference.
Rockville, MD. 2009; [Retrieved: 11 November, 2010]. Available from: http://www.ahrq.gov/about/annualconf09/shiffman.htm

[29] Middleton B. The clinical decision support consortium. Stud Health Technol Inform 2009; 150: 26-30

[30] Fry EA. (Infrastructure: DDSS and KMR. Socratic Grid Project. 2010; [Retrieved: 11 November, 2010]. Available from: http://ww w.socraticgrid. org/index.php/documentation.html.

[31] Fridsma D. CARE-IT Lab. Arizona State University. 2010; [Retrieved: 11 November, 2010]. Available from: http://care-it.asu. edu

[32] Agency for Healthcare Research and Quality. Structuring Care Recommendations for Clinical Decision Support. AHRQ Publication No. 09-M048. 2009; [Retrieved: 11 November, 2010] Available from: http://pbrn.ahrq.gov/portal/server.pt?open=18\&ob $\mathrm{jID}=910228 \&$ qid $=42777884 \&$ rank $=2 \&$ parentname $=$ Community $\mathrm{Pa}$ ge \&parentid $=0 \&$ mode $=2 \&$ in $\_$hi_userid $=8762 \&$ cached $=$ true .

[33] University of Texas Health Science Center at Houston. National Center for Cognitive Informatics and Decision Making in Healthcare. 2010; [Retrieved: 11 November, 2010]. Available from: http://www. uthouston.edu/nccd/projects.htm

[34] Sordo M, Boxwala AA, Ogunyemi O, Greenes RA. Description and status update on GELLO: a proposed standardized objectoriented expression language for clinical decision support. Proc MEDINFO 2004, San Francisco, CA, IMIA: Amsterdam. 2004; 164-168.

[35] RuleML.org. The Rule Markup Initiative. 2010; [Retrieved: 11 November, 2010]. Available from: http://ruleml.org/

[36] Kim S, Haug PJ, Rocha RA, Choi I. Modeling the Arden Syntax for medical decisions in XML. Int J Med Inform 2008; 77(10): 650-6.

(C) Greenes et al.; Licensee Bentham Open.

This is an open access article licensed under the terms of the Creative Commons Attribution Non-Commercial License (http://creativecommons.org/licenses/by-nc 13.0/) which permits unrestricted, non-commercial use, distribution and reproduction in any medium, provided the work is properly cited. 